PREHOSPITAL CARE

\title{
Prehospital management of lower limb fractures
}

\author{
C Lee, K M Porter
}

Emerg Med J 2005;22:660-663. doi: 10.1136/emj.2005.024489

Lower limb fractures are common injuries in prehospital care. Untreated fractures can lead to hypovolaemic shock especially if open, and should be treated with effective haemorrhage control and splintage. A brief assessment for open fractures, deformity, and neurovascular compromise should be followed by effective analgesia, wound management, reduction (if needed), splintage, and packaging of the patient. Early appropriate management reduces the morbidity and mortality of lower limb fractures.

$\mathrm{E}$ xtremity fractures are commonly seen in prehospital care. They demonstrate a wide variety of injury patterns which depend on the patient's age, mechanism of injury, and premorbid pathology.

Road traffic collisions are associated with a number of common fracture patterns-for example, front seat occupants may sustain patella fractures, femoral shaft fractures, and posterior hip dislocations following impact with the dashboard. Intrusion into the foot well may produce ankle fractures or mid-foot fracture dislocations. When struck by a car pedestrians have injuries that reflect whether they were struck from the front or the side-for example, tibial plateau fractures on the side of impact. The motorcyclist in collision with a car may have injuries from the impact with the vehicle, the ground, or surrounding objects-for example, street furniture.

Sporting injuries, especially football tackles, may result in ankle or tibial fractures. Falls from a height may cause multiple fractures in the lower limbs including fractures of the os calcis, fractures of the tibial plafond, tibial plateau fractures, fractures of the shaft of the femur, and acetabular fractures. Even low energy injuries such as tripping over on an uneven surface can cause a complicated fracture dislocation of the ankle.

Patients at risk of pathological fractures-for example, secondary carcinoma or myeloma-may suffer fractures with minimal or no trauma.

It is therefore imperative that the prehospital provider follows a simple logical system to effectively manage lower limb fractures and avoid potential complications (see fig 1 ).

\section{Primary survey}

After scene safety the assessment and management should follow the ABCDE principles. ${ }^{1}$ All patients with significant lower limb fractures should receive high flow oxygen at $15 \mathrm{l} / \mathrm{min}$ via a non-rebreathing trauma mask with a reservoir bag. The management of life threatening injuries that jeopardise airway and breathing should take priority over extremity fractures. The exception to this is exsanguinating external haemorrhage where delays performing assessment of airway and breathing would put the patient at risk of death from blood loss. Recognition of non-immediately life threatening external haemorrhage and its control are important and are usually identified during the circulatory assessment. Lesser bleeding will usually be recognised under the exposure component of the primary survey and minor wounds, often as part of the secondary survey (see table 1).

Untreated fractures of the lower limbs can lead to significant blood loss, which may be external and obvious, or covert. The estimated blood loss for a closed fracture of the femur is $1000-1500 \mathrm{ml}$ and for a closed fracture of the tibia is $500-1000 \mathrm{ml}$. These figures can be doubled if the fracture is open. Fractures of the lower limb, particularly the femur, should be considered a potential cause of hypovolaemic shock, especially if compound.

Control of external haemorrhage should precede fluid resuscitation. Unless haemorrhage is catastrophic (in which

PRIMARY SURVEY WITH ARREST OF HAEMORRHAGE (see table 1)
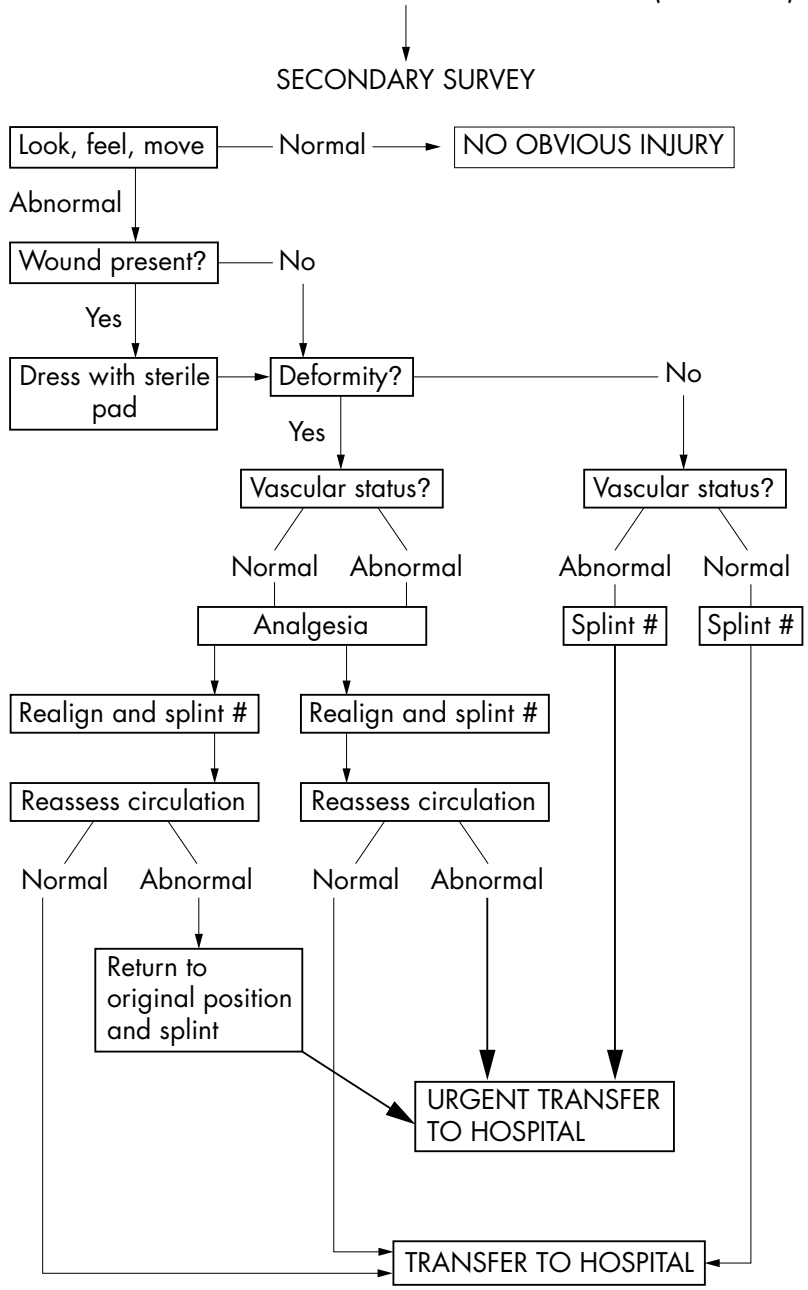

Figure 1 Algorithm for the prehospital management of lower limb fractures. 


\begin{tabular}{ll}
$\begin{array}{l}\text { Table } 1 \text { Assessment of haemorrhage in lower limb } \\
\text { fractures }\end{array}$ \\
\hline Massive exsanguinating haemorrhage & $\mathrm{Cm}^{*} \mathrm{ABCDE}$ \\
Non-exsanguinating significant bleeding & $\mathrm{ABCDE}$ \\
Lesser bleeding & $\mathrm{ABCDE}$ \\
Minor wounds & Secondary survey \\
\hline *Control of massive haemorrhage. & \\
\hline
\end{tabular}

case a tourniquet should be used), control of bleeding should follow a stepwise progression:
- direct pressure
- elevation
- wound packing
- windlass technique
- indirect pressure
- tourniquet.

If, despite direct pressure and elevation, blood soaks through the dressing it should be removed, the wound packed with a dressing, and secured in place as firmly as possible. If blood continues to soak through, a windlass technique can be used to secure haemostasis: a dressing is held in place by a broad bandage (or crepe bandage), another broad bandage is secured with the knot over the wound, and a pen or similar object is placed under the knot and rotated until tight and then secured in place. Although painful when tight it will normally arrest haemorrhage. The neurovascular status of the limb should be assessed following application. If a foot pulse cannot be felt the windlass should be released until the pulse is felt. If rebleeding occurs the dressing can be tightened again to control haemorrhage. In such circumstances it will be necessary to accept that the windlass needs to function as a tourniquet to be lifesaving. The time of application should be noted and the patient transferred to hospital immediately, recognising the presence of a time critical injury. If this technique still proves to be ineffective in arresting haemorrhage a proximal tourniquet should be applied.

A simple and important technique in reducing blood loss involves traction and the splintage of fractures. In the case of untreated femoral shaft fractures, bony overlap and large open venous channels in muscle potentiate bleeding. The application of traction helps realign the limb and closes the venous channels; in effect it reduces the space for bleeding from a sphere (of greater volume) to that of a cylinder. In the case of femoral fractures this is best achieved following titrated opiate analgesia and the position maintained by the application of a traction splint.

The experienced practitioner will be aware that the trauma patient may not exhibit the classical textbook signs of tachycardia and hypotension in hypovolaemic shock. The prehospital provider should be vigilant and prepared for the possibility of circulatory deterioration. The Faculty of Prehospital Care Consensus Statement ${ }^{2}$ recommends that attempts to cannulate the patient should not prolong on scene times and it may be more appropriate to gain intravenous access en route to hospital. The exceptions to this are when the patient is trapped or when vascular access is necessary to provide analgesia.

In the presence of compressible controllable bleeding (most limb fractures) fluid resuscitation is designed to restore normal physiological parameters: pulse and blood pressure. However, in the presence of non-compressible and non-controllable bleeding (some limb fractures) or major bleeding elsewhere (pelvis, chest, abdomen, and reteroperitoneal bleeding), the object of resuscitation should be to maintain essential organ perfusion-the principle of hypotensive resuscitation-maintaining a blood pressure of $80 \mathrm{mmHg}$ or to restore the radial pulse. Fluid should be titrated to response by giving $250 \mathrm{ml}$ aliquots of warmed normal saline. ${ }^{2}$

\section{Secondary survey}

A secondary survey should only be undertaken on completion of the primary survey. Any residual time critical problems from the primary survey may necessitate delay in undertaking the secondary survey which may take place in hospital. As the prehospital provider on scene, it is important to obtain the history of the circumstances surrounding the traumatic episode and to be able to predict the possible injuries: "reading the wreckage".

If the patient is talking, a brief "AMPLE" history needs to be obtained-for example, Allergies, Medication, Past medical history, Last ate, clinical Events about the accident, plus the patient's tetanus status. Should the patient deteriorate in transit this information may be vital. The time of injury should also be recorded.

Examination may be difficult if the patient is trapped, there is poor lighting, or there are difficult environmental conditions. Minimal exposure should occur while the patient is outdoors but a brief assessment for lower limb injury is necessary. If the patient is wearing motorcycle leathers these should be retained to provide a tamponade effect against haemorrhage and soft tissue swelling.

Examination for musculoskeletal injuries should follow the "look, feel, and move" principles of assessment. However, in the presence of obvious fractures it is unnecessary to perform a detailed examination which will produce further pain.

Any open fractures should be identified and an attempt made to remove gross contamination. It may be most appropriate for this procedure to be undertaken on the trolley cot in the back of the ambulance. A polaroid or digital photograph should be taken to provide information for the receiving team and hospital documentation. Gross contamination should be washed or wiped away using saline or saline soak pads and the wound covered with a sterile betadine dressing. Ideally this dressing should not be removed until the patient is in the operating theatre.

The clinical examination should identify deformity of the lower limb which may require manipulation to allow splintage and packaging. The neurovascular status must be assessed before and after patient handling including manipulation. The capillary refill time should be determined and compared to the uninjured limb. Neurovascular deficit mandates immediate realignment which should be undertaken after the provision of adequate analgesia. The limb can normally be realigned by continuous longitudinal traction and manual correction to a neutral position. Repeated neurovascular assessment should then take place. If there has been no improvement the patient should be transported urgently to hospital for definitive care. If attempted reduction has worsened the neurovascular status, the limb should be returned to the original position, splinted, and the patient transported to hospital urgently.

Similarly fracture dislocations should be restored to a normal (or near normal) position as soon as possible particularly if there is a neurovascular deficit or skin compromise. Fracture dislocation of the ankle is the most commonly affected joint. Reduction should only be undertaken if the rescuer has been adequately trained in this procedure. Alternatively the limb should be supported in a padded box splint or equivalent and transported urgently to hospital. 


\section{Analgesia}

There are several choices of analgesia for the prehospital management of lower limb fractures. For patients with isolated tibia or ankle fractures, inhaled nitrous oxide/oxygen (entonox) may be useful for manipulation, splintage, and transfer. ${ }^{3}$ It is contraindicated in patients with potential pnueumothorax or head injury, or where cutting equipment is used for extrication in view of the combustible oxygen content. It should not be used in patients with circulatory shock because it consists of a 50/50 mixture of nitrous oxide and oxygen.

Intravenous morphine provides very effective analgesia, and should be titrated to clinical effect and given with an antiemetic. Morphine is associated with vomiting, hypotension, respiratory depression, and a reduced conscious level in significant doses. In recent years ketamine has become a useful prehospital intravenous analgesic at low dosage $(0.5 \mathrm{mg} / \mathrm{kg}) .^{4}$ This drug should be titrated to response, because in larger doses ketamine acts as an anaesthetic agent. Potential advantages of ketamine include the fact that it does not produce respiratory depression or hypotension and in analgesic doses produces a mild bronchodilator effect. The main disadvantage is an emergence delirium in a small number of patients, which can be treated with $0.5-2 \mathrm{mg}$ of intravenous midazolam.

Peripheral nerve blocks may also be a valid option alone or in conjunction with intravenous analgesia. The most commonly used block in lower limbs is the femoral nerve block, involving injection of local anaesthetic $1-2 \mathrm{~cm}$ lateral to the femoral pulse just below the inguinal ligament. The neurovascular status of the limb must be carefully assessed before the procedure because of the anatomical sensory deficit in the femoral nerve distribution afterwards. There are few immediate complications: the most common is femoral artery puncture which must be identified immediately before injection by withdrawing the plunger of the syringe. If accidental arterial puncture occurs the needle should be removed and firm pressure applied for five minutes, then a more lateral insertion used for further attempts.

Other nerve blocks-for example, ulnar and median nerve-may be used but only by those practitioners with the clinical competence and experience to do so. They may be useful for use in the entrapped patient, for example in machinery.

Regional blocks should not delay the on scene time in the cases of non-entrapment where titrated opiates may be the analgesia of choice.

\section{Splintage}

The application of a splint is an essential aspect of the management of lower limb fractures. The benefits of splintage include reducing pain, reducing blood loss, reducing pressure on skin, reducing pressure on adjacent neurovascular structures, reducing the risk of fat embolism, and reducing the risk of further damage.

The principles of immobilisation include:

- Assessment and reassessment the neurovascular status before and after any manipulation or handling of the fracture.

- Immobilisation of the joints above and below the fracture.

Splints commonly used by the ambulance service include Box splints, Vacuum splints, and Traction splints

\section{Box splints}

These consist of three long padded boards and a foot piece. The patient's shoe and sock are normally removed to facilitate neurovascular assessment and the leg placed in a splint. The foot piece is designed to hold the ankle at right angles. The outer sides of the splint are folded around the leg and secured with Velcro straps. They are suitable for immobilising ankle and tibial fractures, and also injuries around the knee. The splints are available in two appropriate sizes: short leg and long leg.

\section{Vacuum splints}

Vacuum splints provide rigid support and can conform to a deformed limb. Essentially the splint consists of a strong tough plastic material which contains polystyrene beads. Removal of the air from the bag makes the splint rigid. These splints are at risk of puncture and loss of immobilisation particularly if used where there are sharp edges as in vehicle damage.

\section{Traction splints}

Traction splints can be used for closed and open fractures of the shaft of the femur and tibia. The traction splint is used to secure the leg in an improved and near reduced position. The traction principle involves securing the ankle and applying traction by distracting the device with the proximal end of the splint pressing against the pelvis (separating two fixed points). Without traction the splint can also be used to support injuries around the knee. Contra-indications to their use include dislocation of the hip, fracture dislocation of the knee, and ankle injuries.

The types of traction splint include the Donway splint (SP Services, UK), Trac3 splint (Emsar, Taiwan), and the Sager splint (Sager/Minto, California, USA). The attraction of the latter is that it can be used to immobilise both legs (bilateral femoral fractures) simultaneously.

Before and after application the rescuer should check the distal neurovascular status of the limb. The techniques for application are similar and the rescuer should familiarise themself with the splint used by their local ambulance service including its application and removal. An exchange splint should be retained in the accident and emergency department.

The choice of splints depends on availability and injury pattern. Their application is summarised in table 2 .

\begin{tabular}{|c|c|}
\hline Injury & Splintage options \\
\hline Fractured neck of femur & $\begin{array}{l}\text { Padding between legs } \\
\text { Figure of eight bandage around ankles } \\
\text { Broad bandage: two above, two below the } \\
\text { knee }\end{array}$ \\
\hline \multirow{3}{*}{$\begin{array}{l}\text { Fractured shaft of femur } \\
\text { Fracture or fracture } \\
\text { dislocation around the knee }\end{array}$} & Traction splint \\
\hline & $\begin{array}{l}\text { Long leg box splint } \\
\text { Vacuum splint }\end{array}$ \\
\hline & $\begin{array}{l}\text { Traction splint without the application of } \\
\text { traction }\end{array}$ \\
\hline \multirow[t]{5}{*}{ Patella dislocation } & Pre-reduction \\
\hline & $\begin{array}{l}\text { Companion strapping (one leg to the other) } \\
\text { Support on pillow }\end{array}$ \\
\hline & Contoured vacuum splint \\
\hline & Post reduction \\
\hline & Box splint \\
\hline \multirow[t]{3}{*}{ Tibial shaft fracture } & Long leg box splint \\
\hline & Long vacuum splint \\
\hline & $\begin{array}{l}\text { Traction splints particularly useful in the } \\
\text { presence of ipsi lateral tibial and femoral } \\
\text { diaphyseal fractures }\end{array}$ \\
\hline \multirow[t]{2}{*}{ Ankle fracture } & Short leg box splint \\
\hline & Short vacuum splint \\
\hline \multirow[t]{2}{*}{ Foot fractures } & Short box splint \\
\hline & Short vacuum splint \\
\hline
\end{tabular}




\section{SPECIAL SITUATIONS}

\section{Crush injury}

The degree of crush injury is related to the magnitude and duration of crush. Muscle damage leads to hyperkalaemia, rhabdomyolysis and myoglobinaemia, and hypovolaemia. In the presence of crush injury intravenous fluid replacement should be considered early. In isolated crush injury to the limbs, a policy of hypotensive resuscitation should (in the absence of cardiovascular pathology) be replaced by copious crystalloid administration.

\section{Compartment syndrome}

This is commonly seen in significant closed fractures but can also be seen in open fractures. It is related to bleeding or swelling within a close fascial space. The syndrome does take several hours to develop but can seen and diagnosed in the prehospital phase where there is delay to hospital because of entrapment or long transport times.

The clinical features include pain (despite analgesia), extreme pain when moving the toes, and parasthesiae, with pallor and pulselessness as a late feature. Compartment syndrome is a time dependant, limb threatening emergency.

\section{Fat embolism}

The incidence of fat embolism syndrome can be reduced by prompt correction of hypoxia and hypovolaemia and also by effective early fracture immobilisation.

\section{Transport}

Where options exist and the general condition of the patient permits, the patient should be transported to the hospital best suited to their clinical needs. For example, an open fracture with significant soft tissue injury should be conveyed to a hospital providing orthopaedic and plastic surgical services. Depending on local services and protocol this may involve transportation by air ambulance. ${ }^{5}$

\section{CONCLUSION}

A timely and systematic approach to lower limb assessment and management will permit early recognition and management of life and limb threatening injuries. Appropriate wound management and splintage will facilitate patient packaging. The patient should be transported to the hospital best suited for their clinical needs. Optimal management will reduce both the morbidity and mortality of lower limb fractures.

\section{Authors' affiliations}

C Lee, Accident \& Emergency, Manchester Royal Infirmary, Manchester, UK

K Porter, Trauma \& Orthopaedics, Selly Oak Hospital, Birmingham, UK Competing interests: none declared

Correspondence to: Mr K M Porter, Consultant Trauma \& Orthopaedic Surgeon, Selly Oak Hospital, Raddlebarn Road, Selly Oak, Birmingham B29 6JD, UK; keith.porter@uhb.nhs.uk

Accepted 11 April 2005

\section{REFERENCES}

1 American College of Surgeons Committee on Trauma. In: ATLS manual, 6th edn. Chicago: American College of Surgeons 1997.

2 Revell M, Porter K, Greaves I. Fluid resuscitation in prehospital trauma care: a consensus view. EMJ 2002;19:494-8.

3 O'Sullivan I, Benger J. Nitrous oxide in emergency medicine. EMJ 2003;20:214-17.

4 Porter K. Ketamine in prehospital care. EMJ 2004;21:351-4.

5 Black JJM, Ward ME, Lockey DJ. Appropriate use of helicopters to transport trauma patients from incident scene to hospital in the United Kingdom: an algorithm. EMJ 2004;21:355-61. 